\title{
STABILITY OF LINEAR IMPULSIVE DIFFERENTIAL EQUATIONS VIA $t_{\infty}$-SIMILARITY
}

\author{
Sung Kyu Choi*, Namjip Koo**, and Chunmi Ryu***
}

\begin{abstract}
In this paper we investigate $h$-stability for linear impulsive equations using the notion of $t_{\infty}$-similarity and an impulsive integral inequality.
\end{abstract}

\section{Introduction}

The impulsive differential equations describe evolution process which at certain moments change their state rapidly. In the mathematical simulation of such processes it is convenient to assume that this change takes place momentarily and the process changes its state by jump. Thus, the impulsive differential equations are adequate mathematical models for description of evolution processes characterized by the combination of a continuous and jump change of their states. It is now being recognized that the theory of impulsive differential equations is not only richer than the corresponding theory of differential equations but also represents a more natural framework for mathematical modelling of many real world phenomena. For a detail discussion of impulsive integral inequalities and some basic concepts concerning about the impulsive differential equations, we refer the reader to $[1,2,7]$.

The notion of $h$-stability for differential equations was introduced by Pinto and includes several types of known stability properties as uniform stability, uniform Lipschitz stability and exponential asymptotic stability $[8,9]$.

Received August 20, 2013; Accepted September 27, 2013.

2010 Mathematics Subject Classification: Primary 34A37, 34D20.

Key words and phrases: impulsive differential equations, impulsive integral inequalities, $h$-stability, $t_{\infty}$-similarity.

Correspondence should be addressed to Namjip Koo, njkoo@cnu.ac.kr.

This work was supported by Basic Science Research Program through the National Research Foundation of Korea(NRF) funded by the Ministry of Education(NRF2013R1A1A2007585). 
Choi et al. studied $h$-stability for the nonlinear Volterra integrodifferential system [4] and nonlinear perturbed systems [3]. Moreover, the notion of $t_{\infty}$-similarity and Liapunov functions were used to study $h$-stability for nonlinear differential systems [5].

In this paper we study $h$-stability for linear impulsive equations using the notion of $t_{\infty}$-similarity and an impulsive integral inequality.

\section{Main results}

Suppose that $\left(\tau_{k}\right) \subset \mathbb{R}$ is a fixed sequence and satisfies the condition

$$
\tau_{k}<\tau_{k+1}, k \in \mathbb{Z} \text { and } \lim _{k \rightarrow \pm \infty} \tau_{k}= \pm \infty .
$$

Let $P C\left(\mathbb{R}, \mathbb{R}^{n \times n}\right)$ be the set of functions $\psi: \mathbb{R} \rightarrow \mathbb{R}^{n \times n}$ which are continuous for $t \in \mathbb{R}, t \neq \tau_{k}$, are continuous from the left for $t \in \mathbb{R}$, and have discontinuities of the first kind at the points $\tau_{k} \in \mathbb{R}$ for each $k \in \mathbb{Z}$.

We consider two linear homogeneous impulsive equations

$$
\left\{\begin{aligned}
x^{\prime} & =A(t) x, \quad t \neq \tau_{k}, \\
\Delta x & =A_{k} x, \quad t=\tau_{k}, \quad k \in \mathbb{Z},
\end{aligned}\right.
$$

and

where

$$
\left\{\begin{aligned}
y^{\prime} & =B(t) y, \quad t \neq \tau_{k}, \\
\Delta y & =B_{k} y, \quad t=\tau_{k}, \quad k \in \mathbb{Z},
\end{aligned}\right.
$$

$$
A, B \in P C\left(\mathbb{R}, \mathbb{R}^{n \times n}\right), A_{k}, B_{k} \in \mathbb{R}^{n \times n}, \operatorname{det}\left(E+B_{k}\right) \neq 0, k \in \mathbb{Z} .
$$

Also, we consider the perturbed nonlinear homogeneous impulsive equation of $(2.2)$

$$
\left\{\begin{array}{c}
y^{\prime}=A(t) y+g(t, y), \quad t \neq \tau_{k}, g(t, 0)=0, \\
\Delta y=A_{k} y+g_{k}(y), \quad t=\tau_{k}, g_{k}(0)=0, k \in \mathbb{Z},
\end{array}\right.
$$

where $g \in C_{\tau}\left(\mathbb{R} \times \mathbb{R}^{n}, \mathbb{R}^{n}\right)$ and $g_{k} \in C\left(\mathbb{R}^{n}, \mathbb{R}^{n}\right)$ for each $k \in \mathbb{Z}$, respectively.

Lemma 2.1. [2, Theorem 1.5] Let conditions (2.1) and (2.4) hold. Then the following statements hold:

(1) For any $\left(t_{0}, x_{0}\right) \in \mathbb{R} \times \mathbb{R}^{n}$, there exists a unique solution of equation (2.2) with $x\left(t_{0}^{+}\right)=x_{0}$ (or $x\left(t_{0}\right)=x_{0}$ ) and this solution is defined for $t>t_{0}\left(\right.$ or $\left.t \geq t_{0}\right)$.

(2) If $\operatorname{det}\left(E+A_{k}\right) \neq 0$ for each $k \in \mathbb{Z}$, then this solution is defined for all $t \in \mathbb{R}$. 
We can obtain the following result under a suitable condition from Theorem 1.11 in [2].

Lemma 2.2. [2] Each solution $y(t)=y\left(t, t_{0}^{+}, y_{0}\right)$ of (2.5) with $y\left(t_{0}^{+}\right)=$ $y_{0}$ satisfies the integro-summary equation

$$
\begin{aligned}
y(t)= & X\left(t, t_{0}^{+}\right) y_{0}+\int_{t_{0}}^{t} X(t, \tau) g(\tau, y(\tau)) d \tau \\
& +\sum_{t_{0} \leq \tau_{k}<t} X\left(t, \tau_{k}^{+}\right) g_{k}\left(y\left(\tau_{k}\right)\right), t \geq t_{0}^{+}, k \in \mathbb{Z},
\end{aligned}
$$

where $X(t)$ is a fundamental matrix of (2.2) and $X\left(t, t_{0}\right) \equiv X(t) X^{-1}\left(t_{0}\right)$.

Lemma 2.3. [2, Lemma 1.4] Suppose that for $t \geq t_{0}$ the inequality

$$
u(t) \leq c+\int_{t_{0}}^{t} b(s) u(s) d s+\sum_{t_{0} \leq \tau_{k}<t} \beta_{k} u\left(\tau_{k}\right)
$$

holds, where $u \in P C\left(\mathbb{R}, \mathbb{R}^{+}\right), b \in P C\left(\mathbb{R}, \mathbb{R}^{+}\right), c$ and $\beta_{k}$ are nonnegative constants for each $k \in \mathbb{Z}$. Then we have

$$
\begin{aligned}
u(t) & \leq c \prod_{t_{0} \leq \tau_{k}<t}\left(1+\beta_{k}\right) \exp \left(\int_{t_{0}}^{t} b(s) d s\right) \\
& \leq c \exp \left(\int_{t_{0}}^{t} b(s) d s+\sum_{t_{0} \leq \tau_{k}<t} \beta_{k}\right), t \geq t_{0}, k \in \mathbb{Z} .
\end{aligned}
$$

REMARK 2.4. If $A(t)$ and $B(t)$ are similar, i.e., there exists an invertible bounded matrix $S(t)$ with bounded $S^{-1}(t)$ such that $S A S^{-1}=B$, then $\exp (A t)$ and $\exp (B t)$ are also similar.

We recall the notion of $h$-stability for impulsive differential equations.

Definition 2.5. [6] The zero solution $x=0$ of (2.5) is called $h$-stable if there exist a positive bounded left continuous function $h: \mathbb{R}^{+} \rightarrow \mathbb{R}$ and a constant $c \geq 1$ such that

$$
\left|x\left(t, t_{0}, x_{0}\right)\right| \leq c\left|x_{0}\right| h(t) h\left(t_{0}\right)^{-1}, t \geq t_{0},
$$

for $\left|x_{0}\right|$ small enough (here $h(t)^{-1}=\frac{1}{h(t)}$ ).

We need the following lemma for $h$-stability of solutions of linear impulsive differential systems.

Lemma 2.6. [9, Lemma 1] The linear impulsive equation (2.2) is $h$ stable if and only if there exist a constant $c \geq 1$ and a positive bounded left continuous function $h: \mathbb{R}^{+} \rightarrow \mathbb{R}$ such that for every $x_{0} \in \mathbb{R}^{n}$,

$$
\left|X\left(t, t_{0}\right)\right| \leq \operatorname{ch}(t) h\left(t_{0}\right)^{-1}, t \geq t_{0} \geq 0,
$$


where $X(t)$ is a fundamental matrix of (2.2).

We improve the result of Theorem 2.7 in [6] for nonlinear impulsive differential equations.

Theorem 2.7. Suppose that the perturbed terms $g$ and $g_{k}$ of (2.5) satisfy the following conditions:

$$
\begin{aligned}
|g(t, y)| & \leq L d(t)|y|, \\
\left|g_{k}(y)\right| & \leq d_{k}|y|, k \in \mathbb{Z},
\end{aligned}
$$

where $d \in P C\left(\mathbb{R}, \mathbb{R}^{+}\right), d_{k} \in \mathbb{R}^{+}, L$ is a nonnegative constant, and there exists a positive constant $M$ such that

$$
c L \int_{0}^{\infty} d(s) d s+c \sum_{0 \leq \tau_{k} \leq \infty} \frac{h\left(\tau_{k}\right)}{h\left(\tau_{k}^{+}\right)} d_{k} \leq M .
$$

If the zero solution $x=0$ of (2.2) is $h$-stable, then the zero solution $y=0$ of $(2.5)$ is $h$-stable.

Proof. Let $y(t)=y\left(t, t_{0}, y_{0}\right)$ be any solution of (2.5) with $y\left(t_{0}\right)=y_{0}$. Since the zero solution $x=0$ of $(2.2)$ is $h$-stable, Then, from Lemma 2.6 , there exist a constant $c \geq 1$ and a positive bounded left continuous function $h: \mathbb{R}^{+} \rightarrow \mathbb{R}$ such that

$$
\left|X\left(t, t_{0}\right)\right| \leq \operatorname{ch}(t) h\left(t_{0}\right)^{-1}, t \geq t_{0},
$$

where $X(t)$ is a fundamental matrix for (2.2). Then it follows from Lemmas 2.2 and 2.6 that

$$
\begin{aligned}
|y(t)| \leq & \left|X\left(t, t_{0}\right)\right|\left|y_{0}\right|+L \int_{t_{0}}^{t}|X(t, \tau)| d(\tau)|y(\tau)| d \tau \\
& +\sum_{t_{0} \leq \tau_{k}<t}\left|X\left(t, \tau_{k}^{+}\right)\right| d_{k}\left|y\left(\tau_{k}\right)\right| \\
\leq & c h(t) h\left(t_{0}\right)^{-1}\left|y_{0}\right|+c L \int_{t_{0}}^{t} h(t) h(\tau)^{-1} d(\tau)|y(\tau)| d \tau \\
& +c \sum_{t_{0} \leq \tau_{k}<t} h(t) h\left(\tau_{k}^{+}\right)^{-1} d_{k}\left|y\left(\tau_{k}\right)\right|, t \geq t_{0} .
\end{aligned}
$$

Letting $u(t)=\frac{|y(t)|}{h(t)}$, we have

$$
u(t) \leq c u\left(t_{0}\right)+c L \int_{t_{0}}^{t} d(s) u(s) d s+c \sum_{t_{0} \leq \tau_{k}<t} \frac{h\left(\tau_{k}\right)}{h\left(\tau_{k}^{+}\right)} d_{k} u\left(\tau_{k}\right), t \geq t_{0} .
$$

By Lemma 2.3, we obtain 


$$
\begin{aligned}
|y(t)| & \leq c h(t) h\left(t_{0}\right)^{-1}\left|y_{0}\right| \exp \left(c L \int_{t_{0}}^{t} d(s) d s+c \sum_{t_{0} \leq \tau_{k}<t} \frac{h\left(\tau_{k}\right)}{h\left(\tau_{k}^{+}\right)} d_{k}\right) \\
& \leq c h(t) h\left(t_{0}\right)^{-1}\left|y_{0}\right| \exp \left(c L \int_{t_{0}}^{\infty} d(s) d s+c \sum_{t_{0} \leq \tau_{k}<\infty} \frac{h\left(\tau_{k}\right)}{h\left(\tau_{k}^{+}\right)} d_{k}\right) \\
& \leq \hat{c}\left|y\left(t_{0}\right)\right| h(t) h\left(t_{0}\right)^{-1}, t \geq t_{0},
\end{aligned}
$$

where $\hat{c}=c \exp (M)$. Hence the zero solution $y=0$ of $(2.5)$ is $h$-stable. This completes the proof.

REMARK 2.8. We note that if $h(t)$ in Theorem 2.7 is continuous, then we have $\frac{h\left(\tau_{k}\right)}{h\left(\tau_{k}^{+}\right)}=1$ for each $k \in \mathbb{Z}$.

We can obtain the following result as the corollary of Theorem 2.7.

Corollary 2.9. [6, Theorem 2.7] Suppose that the perturbed terms $g$ and $g_{k}$ of (2.5) satisfy the following conditions:

$$
\begin{aligned}
g(t, y) & =D(t) y, \\
g_{k}(y) & =D_{k} y, k \in \mathbb{Z},
\end{aligned}
$$

where $D \in P C\left(\mathbb{R}, \mathbb{R}^{n \times n}\right), D_{k} \in \mathbb{R}^{n \times n}$, and $\operatorname{det}\left(E+A_{k}+D_{k}\right) \neq 0$ for each $k \in \mathbb{Z}$. Assume that there exists a positive constant $M$ such that

$$
c \int_{0}^{\infty}|D(s)| d s+c \sum_{0 \leq \tau_{k} \leq \infty} \frac{h\left(\tau_{k}\right)}{h\left(\tau_{k}^{+}\right)}\left|D_{k}\right| \leq M .
$$

If the zero solution $x=0$ of (2.2) is $h$-stable, then the zero solution $y=0$ of (2.5) is h-stable.

Example 2.10. [6, Example 2.10]To illustrate Lemma 2.6, we consider the linear impulsive differential equation

$$
\left\{\begin{aligned}
x^{\prime}(t) & =a(t) x, t \neq \tau_{k}, \\
\Delta x & =a_{k} x, \quad t=\tau_{k}, k \in \mathbb{Z},
\end{aligned}\right.
$$

where $a \in P C(\mathbb{R}, \mathbb{R}), a_{k} \in \mathbb{R}$, and $\operatorname{det}\left(1+a_{k}\right) \neq 0$ for each $k \in \mathbb{Z}$. Suppose that $\int_{t_{0}}^{\infty}|a(s)| d s<\infty$ and $\sum_{t_{0} \leq \tau_{k} \leq \infty}\left|a_{k}\right|<\infty$ for each $t_{0} \in \mathbb{R}$. Then the zero solution $x=0$ of (2.12) is $h$-stable.

Let $\mathcal{S}$ be the set of all matrix functions $S: \mathbb{R}^{+} \rightarrow \mathbb{R}^{n \times n}$ which belong to $P C\left(\mathbb{R}^{+}, \mathbb{R}^{n \times n}\right)$ and are bounded in $\mathbb{R}^{+}$together with inverse $S^{-1}(t)$. Let $\mathcal{M}$ be denoted by

$\mathcal{M}=\left\{\left(A, A_{k}\right) \mid A \in P C\left(\mathbb{R}^{+}, \mathbb{R}^{n \times n}\right), A_{k} \in \mathbb{R}^{n \times n}, \operatorname{det}\left(E+A_{k}\right) \neq 0, k \in \mathbb{N}\right\}$. 
Definition 2.11. We say that $\left(A, A_{k}\right) \in \mathcal{M}$ is $t_{\infty}$-similar to $\left(B, B_{k}\right) \in$ $\mathcal{M}$ if there exists a matrix function $S \in \mathcal{S}$ such that

$$
\begin{gathered}
S^{\prime}(t)-A(t) S(t)+S(t) B(t) \equiv F_{0} \in L_{1}, t \neq \tau_{k}, \\
\Delta S\left(\tau_{k}\right)-A_{k} S\left(\tau_{k}\right)+S\left(\tau_{k}^{+}\right) B_{k} \equiv F_{0 k} \in l_{1}, t=\tau_{k}, k \in \mathbb{N},
\end{gathered}
$$

where $\Delta S\left(\tau_{k}\right)=S\left(\tau_{k}^{+}\right)-S\left(\tau_{k}\right)$. We can say that equations (2.2) and (2.3) are $t_{\infty}$-similar if $\left(A, A_{k}\right) \in \mathcal{M}$ is $t_{\infty}$-similar to $\left(B, B_{k}\right) \in \mathcal{M}$ for each $k \in \mathbb{N}$. Note that the relation $\left(A, A_{k}\right) \sim\left(B, B_{k}\right): S$ is an equivalence relation on $\mathcal{M}[2]$.

REMARK 2.12. The notion of $t_{\infty}$-similarity preserves various stability concepts: stability, uniform stability, uniform asymptotic stability, strict stability [2, Theorem 10.3]. In particular, when $F_{0}=0$ in (2.13) and $F_{0 k}=0$ in (2.14) for each $k \in \mathbb{N}$, then $t_{\infty}$-similarity implies kinematical similarity.

Lemma 2.13. Suppose that equations (2.2) and (2.3) are $t_{\infty}$-similar. Then we have

$$
\begin{aligned}
S(t)= & X(t)\left[X^{-1}(\tau) S(\tau) Y(\tau)+\int_{\tau}^{t} X^{-1}(s) F_{0}(s) Y(s) d s\right. \\
& \left.+\sum_{\tau \leq \tau_{k}<t} X^{-1}\left(\tau_{k}^{+}\right) F_{0 k} Y\left(\tau_{k}\right)\right] Y^{-1}(t), t \geq \tau \geq t_{0}, k \in \mathbb{N},
\end{aligned}
$$

where $X(t)$ and $Y(t)$ are fundamental matrices of (2.2) and (2.3), respectively.

Proof. From a simple calculation, we obtain

$$
\begin{aligned}
(S Y)^{\prime} & =S^{\prime} Y+S Y^{\prime} \\
& =\left[F_{0}+A S-S B+S B\right] Y \\
& =A(S Y)+F_{0} Y, t \neq \tau_{k},
\end{aligned}
$$

and

$$
\begin{aligned}
\Delta(S Y)_{k} & =\Delta S_{k} Y_{k}+S_{k} \Delta Y_{k} \\
& =\left[F_{0 k}+A_{k} S_{k}-S_{k}^{+} B_{k}+S_{k}^{+} B_{k}\right] Y_{k} \\
& =A_{k}(S Y)_{k}+F_{0 k} Y_{k}, t=\tau_{k}, k \in \mathbb{N} .
\end{aligned}
$$

Hence we have

$$
\begin{aligned}
Y(t)= & S^{-1}(t) X(t)\left[X^{-1}(\tau) S(\tau) Y(\tau)+\int_{\tau}^{t} X^{-1}(s) F_{0}(s) Y(s) d s\right. \\
& \left.+\sum_{\tau \leq \tau_{k}<t} X^{-1}\left(\tau_{k}^{+}\right) F_{0 k} Y\left(\tau_{k}\right)\right], t \geq \tau \geq t_{0}, k \in \mathbb{N} .
\end{aligned}
$$


This completes the proof.

The results of various stabilities in Theorem 10.3 in [2] are generalized for impulsive linear equations using $t_{\infty}$-similarity.

Theorem 2.14. Suppose that equations (2.2) and (2.3) are $t_{\infty}$-similar and $\sup _{k \in \mathbb{N}} \frac{h\left(\tau_{k}\right)}{h\left(\tau_{k}^{+}\right)}$is bounded. Then the solution $x=0$ of $(2.2)$ is $h$-stable if and only if the solution $y=0$ of (2.3) is h-stable.

Proof. Suppose that the solution $x=0$ of $(2.2)$ is $h$-stable. Then there exist a constant $c_{1} \geq 1$ and a positive bounded left continuous functions $h$ defined on $\mathbb{R}^{+}$such that

$$
\left|X\left(t, t_{0}\right)\right| \leq c_{1} h(t) h\left(t_{0}\right)^{-1}, t \geq t_{0} \geq 0,
$$

where $X(t)$ is a fundamental matrix of (2.2). By Lemma 2.13, we have

$$
\begin{aligned}
Y(t)= & S^{-1}(t) X(t)\left[X^{-1}(\tau) S(\tau) Y(\tau)+\int_{\tau}^{t} X^{-1}(s) F_{0}(s) Y(s) d s\right. \\
& \left.+\sum_{\tau \leq \tau_{k}<t} X^{-1}\left(\tau_{k}^{+}\right) F_{0 k} Y\left(\tau_{k}\right)\right], t \geq \tau \geq t_{0}, k \in \mathbb{N},
\end{aligned}
$$

where $Y(t)$ is a fundamental matrix of (2.3). Then from (2.15) and by virtue of the boundedness of $S(t)$ and $S^{-1}(t)$ there are positive constants $c_{1}$ and $c_{2}$ such that

$$
\begin{aligned}
|Y(t, \tau)| \leq & \left|S^{-1}(t)\right||X(t, \tau)||S(\tau)|+\int_{\tau}^{t}|X(t, s)|\left|F_{0}(s)\right||Y(s, \tau)| d s \\
& \left.+\sum_{\tau \leq \tau_{k}<t}\left|X\left(t, \tau_{k}^{+}\right)\right|\left|F_{0 k}\right|\left|Y_{k}\right|\left|Y^{-1}(\tau)\right|\right] \\
\leq & c_{1} c_{2} h(t) h(\tau)^{-1}+c_{1} c_{2} \int_{\tau}^{t} h(t) h(s)^{-1}\left|F_{0}(s)\right||Y(s, \tau)| d s \\
& +c_{1} c_{2} \sum_{\tau \leq \tau_{k}<t} h(t) h\left(\tau_{k}^{+}\right)^{-1}\left|F_{0 k}\right|\left|Y\left(\tau_{k}, \tau\right)\right|, t \geq \tau \geq t_{0} .
\end{aligned}
$$

Letting $u(t)=\frac{|Y(t, \tau)|}{h(t)}$, we have

$$
\begin{aligned}
u(t) \leq & c_{1} c_{2} u(\tau)+\int_{\tau}^{t} c_{1} c_{2}\left|F_{0}(s)\right| u(s) d s \\
& \left.+c_{1} c_{2} \sum_{\tau \leq \tau_{k}<t} \frac{h\left(\tau_{k}\right)}{h\left(\tau_{k}^{+}\right)}\left|F_{0 k}\right| u\left(\tau_{k}\right)\right], t \geq \tau \geq t_{0} .
\end{aligned}
$$

From Lemma 2.3, we have 


$$
\begin{aligned}
\frac{|Y(t, \tau)|}{h(t)} \leq & c_{1} c_{2} u(\tau) \exp \left(\int_{\tau}^{t} c_{1} c_{2}\left|F_{0}(s)\right| d s\right. \\
& \left.+c_{1} c_{2} \sum_{\tau \leq \tau_{k}<t} \frac{h\left(\tau_{k}\right)}{h\left(\tau_{k}^{+}\right)}\left|F_{0 k}\right|\right), t \geq \tau \geq t_{0} .
\end{aligned}
$$

Hence we obtain

$$
\begin{aligned}
|Y(t, \tau)| \leq & c_{1} c_{2} h(t) h(\tau)^{-1} \exp \left(\int_{\tau}^{t} c_{1} c_{2}\left|F_{0}(s)\right| d s\right. \\
& \left.+c_{1} c_{2} \sum_{\tau \leq \tau_{k}<t} \frac{h\left(\tau_{k}\right)}{h\left(\tau_{k}^{+}\right)}\left|F_{0 k}\right|\right) \\
\leq & \operatorname{ch}(t) h(\tau)^{-1}, t \geq \tau \geq t_{0}, k \in \mathbb{N},
\end{aligned}
$$

where $c$ is a positive constant given by

$$
c \equiv c_{1} c_{2} \exp \left(\int_{\tau}^{\infty} c_{1} c_{2}\left|F_{0}(s)\right| d s+c_{1} c_{2} \sum_{\tau \leq \tau_{k} \leq \infty} \frac{h\left(\tau_{k}\right)}{h\left(\tau_{k}^{+}\right)}\left|F_{0 k}\right|\right) .
$$

Hence the solution $y=0$ of (2.3) is $h$-stable by Lemma 2.6.

The converse also holds by the similar method. This completes the proof.

We can obtain the following result as a corollary of Theorem 2.14.

Corollary 2.15. [6, Theorem 2.13] Suppose that (2.2) and (2.3) are kinematically similar. Then (2.2) is h-stable if and only if (2.3) is also $h$-stable.

REMARK 2.16. Suppose that (2.2) and (2.3) are $t_{\infty}$-similar.

(1) If we set $h(t)=c$ for a positive constant $c$, then (2.2) is uniformly stable if and only if (2.3) is also uniformly stable.

(2) If we set $h(t)=e^{-\lambda t}$ for a positive constant $\lambda$, then (2.2) is uniformly exponentially stable if and only if (2.3) is also uniformly exponentially stable.

\section{References}

[1] D. D. Bainov and P. S. Simeonov, Systems with Impulsive Effect: Stability, Theory and Applications, Ellis Horwood Series in Mathematics and Its Application, 1989.

[2] D. D. Bainov and P. S. Simeonov, Impulsive Differential Equations: Asymptotic Properties of the Solutions, World Scientific Publishing Co. Inc., River Edge, NJ, 1995. 
[3] S. K. Choi and N. J. Koo, h-Stability for nonlinear perturbed systems, Ann. Differential Equations 11 (1995), 1-9.

[4] S. K. Choi and H. S. Ryu, h-Stability in differential systems, Bull. Inst. Acad. Sinica 21 (1993), 245-262.

[5] S. K. Choi, N. J. Koo, and H. S. Ryu, h-Stability of differential systems via $t_{\infty}$-similarity, Bull. Korean Math. Soc. 34 (1997), 371-383.

[6] S. K. Choi, N. J. Koo, and C. Ryu, h-Stability of linear impulsive differential equations via similarity, J. Chungcheong Math. Soc. 24 (2011), 393-400.

[7] V. Lakshmikantham, D. D. Bainov, and P. S. Simeonov, Theory of Impulsive Differential Equations, World Scientific Publishing Co. Pte. Ltd., Singapore, 1989.

[8] M. Pinto, Perturbations of asymptotically stable differential systems, Analysis 4 (1984), 161-175.

[9] M. Pinto, Stability of nonlinear differential systems, Applicable Analysis 43 (1992), 1-20.

Department of Mathematics

Chungnam National University

Daejeon 305-764, Republic of Korea

E-mail: sgchoi@cnu.ac.kr

**

Department of Mathematics

Chungnam National University

Daejeon 305-764, Republic of Korea

E-mail: njkoo@cnu.ac.kr

$* * *$

Department of Mathematics

Chungnam National University

Daejeon 305-764, Republic of Korea

E-mail: chmiry@yahoo.co.kr 